\title{
Risk factors, antimicrobial susceptibility pattern and patient outcomes of Pseudomonas aeruginosa infection: A matched case-control study
}

\section{Fatima A Alhussain}

Almithnab General Hospital, AlQassim

\section{Fahad A Al Eidan}

King Saud bin Abdulaziz University for Health Sciences College of Nursing at Al-Ahsa

\section{Sameera Al Johani}

College of Applied Medical Sciences

Motasim Badri ( $\sim$ motasimb@hotmail.com )

King Saud bin Abdulaziz University for Health Sciences https://orcid.org/0000-0002-5268-6492

Research article

Keywords: Pseudomonas aeruginosa, infection, risk factors, resistance, intensive care unit

Posted Date: July 4th, 2019

DOI: https://doi.org/10.21203/rs.2.11006/v1

License: (c) (i) This work is licensed under a Creative Commons Attribution 4.0 International License. Read Full License 


\section{Abstract}

Background Pseudomonas aeruginosa (P.aeruginosa) is a leading nosocomial gram-negative pathogen associated with prolonged hospitalization, morbidity and mortality. Limited data exist regarding P.aeruginosa infection and outcome in patients managed in intensive care units (ICUs). We aimed to determine the risk factors, antimicrobial susceptibility pattern and patient outcomes of P.aeruginosa infection. Methods In this matched case-control study, all P.aeruginosa infections that occurred $>48$ hours after hospital admission between January 31st 2016 and December 31st 2018 at ICUs affiliated with King Abdulaziz Medical City, Riyadh were included. P.aeruginosa bacilli was confirmed using API 20E test and antimicrobial susceptibility using disk diffusion or Epsilometer test. Results The study included 90 cases and 90 controls. Compared with controls, cases had significantly higher mean ICU stay days, proportions of patients with previous history of antimicrobial therapy, coronary artery disease, malignancy, hemodialysis, previous surgery, use of central line, urethral catheterization, nasogastric tube, and tracheostomy. In a multivariate conditional logistic regression analysis, factors independently associated with P.aeruginosa infection were ICU duration [Odds Ratio $(\mathrm{OR})=9.05,95 \% \mathrm{Cl}$ 2.53-32.27, $\mathrm{p}=.001]$, previous surgery $(\mathrm{OR}=7.33,95 \% \mathrm{Cl} 1.66-32.36, \mathrm{p}=.009)$, tracheostomy $(\mathrm{OR}=11.13,95 \% \mathrm{Cl} 1.05-$ $118.59, \mathrm{p}=.046)$, urethral catheterization $(\mathrm{OR}=7.38,95 \% \mathrm{Cl} 1.21-45.11, \mathrm{p}=.030)$ and aminoglycoside drug class $(\mathrm{OR}=10.59,95 \% \mathrm{Cl} 1.14-98.13, \mathrm{p}=.038)$. Of antimicrobial drugs used, highest proportion of resistance was calculated for patients receiving tigecycline (93.3\%). Mortality was high in both groups but did not differ significantly: $54(60 \%)$ cases and $51(56.7 \%)$ controls; $p=.650$. Conclusions: The study identifies several modifiable factors associated with P.aeruginosa infection in ICUs. These factors are of paramount relevance for case identification, control measures, and optimal treatment strategies of P.aeruginosa infection in ICUs.

\section{Background}

Pseudomonas aeruginosa ( $P$.aeruginosa) is a gram-negative bacterium that survives various surfaces and environments and produces a wide range of extracellular toxins. P.aeruginosa is one of the most common causative pathogen that contributes significantly to infection, prolonged hospitalization, and increased mortality ${ }^{1-5}$ According to the World Health Organization (WHO), P.aeruginosa is one of the 12 bacteria priority pathogens that pose the greatest threat to human health. ${ }^{6}$ Although $P$.aeruginosa rarely causes serious infections in healthy individuals, it is associated with various invasive infections in patients with long-term hospitalization, critically ill intubated patients, those treated with broad-spectrum antimicrobial therapy or cancer chemotherapy, and patients with ventilator-associated pneumonia. ${ }^{7}$

Resistance of P.aeruginosa to antipseudomonal drugs continues to increase over the years in several regions of the world. Antimicrobial resistance in the ICU contributes significantly to the incidence of $P$.aeruginosa infections within few days of antimicrobial therapy commencement. Furthermore, coinfection with other microorganism prior to the isolation, total parenteral nutrition usage, comorbid cerebrovascular disease, history of cerebrovascular disease, admission to ICU, malignancy, compromised 
immune system, obstruction coronary disease are known risk factors for P.aeruginosa infection. ${ }^{7}$ Mechanical ventilation, acute respiratory failure, infection site in the respiratory tract and central vein catheter, intubation and usage of multiple invasive devices are also implicated.$^{8,9}$ However, $P$. aeruginosa has organism's multiple resistance mechanisms. Consequently, antimicrobial treatment is limited.

In the Gulf countries, few studies exist on P.aeruginosa infection in adult ICUs. These studies show that P.aeruginosa is one of the most commonly isolated pathogens in the ICUs. In a study conducted in eight major hospitals, the majority of P.aeruginosa isolates were from patients suffering from respiratory tract and wound infections. ${ }^{10}$ The highest resistance to antimicrobials was exhibited by meropenem followed by ticarcillin, imipenem, and piperacillin. Overall, P.aeruginosa strains showed high resistance to meropenem followed by ticarcillin and imipenem; approximately $13 \%$ of the studied strains were multidrug resistant. ${ }^{10}$ In another study, the majority of $P$.aeruginosa resistance to imipenem was calculated in patients with hematologic or solid organ malignancies ${ }^{11}$.

As of 1998, higher rates of resistant bacteria have been seen in Saudi Arabia. Most of these cases could be attributed to greater and irrational use of antibiotic drugs. ${ }^{12}$ In a study conducted in a major tertiary hospital, most $P$.aeruginosa strains were isolated from ICUs and male medical wards. P.aeruginosa was most susceptible to imipenem, amikacin, meropenem, and piperacillin/tazobactam. ${ }^{13}$ In a recent study in a large tertiary care center in Riyadh, P.aeruginosa was the most frequently isolated organism. ${ }^{14}$

P.aeruginosa causes substantial burden in terms of morbidity and mortality. In the USA, P.aeruginosa associated mortality rate amounted to $16 \%$ to $80 \% .{ }^{15}$ In a systematic review and meta-analysis, Nathwani et al. found that in-hospital all-cause mortality ranged between $25 \%-60 \%$ in multi-drug resistant (MDR) $P$.aeruginosa cases. Furthermore, all-cause mortality in patients infected with resistant $P$.aeruginosa was $15 \%-59 \%$, and $7 \%-50 \%$ among $P$.aeruginosa susceptible patients ${ }^{16}$

However, risk factors, antimicrobial susceptibility pattern, and outcomes of patients infected with P.aeruginosa have not been adequately characterized, particularly in the Gulf countries. The aim of this study was to determine these aspects of P.aeruginosa in patients managed in ICUs of one of largest tertiary healthcare institution in the Kingdom of Saudi Arabia.

\section{Methods}

This matched case-control study was conducted at King Abdulaziz Medical City (KAMC), Ministry of National Guard (MNG). KAMC is a tertiary care referral hospital in Riyadh, Kingdom of Saudi Arabia. Cases included all patients diagnosed with $P$. aeruginosa between January $31^{\text {st }} 2016$ and December $31^{\text {st }}$ 2018 in the seven adult ICUs in the hospital: AICU (Adult Intensive Care Unit), NCCU (Neuro Critical Care Unit), BICU (Burn Intensive Care Unit), TICU (Trauma Intensive Care Unit), MCICU (Medical Cardiac Intensive Care Unit), SICU (Surgical Intensive Care Unit), AICU2 (Adult Intensive Care Unit-Oncology). 
$P$.aeruginosa was defined as a nosocomial acquired infection that occurred $>48$ hours after hospital admission. $P$.aeruginosa bacilli was identified using an automated system (MicroScan Walkaway, Siemens) and confirmed using API 20E, and the antimicrobial susceptibility testing was performed using an automated system (MicroScan Walkaway, Siemens) and confirmed by the disk diffusion method or ETest (AB Biodisk). ${ }^{18}$ All tests were performed according to The USA National Committee for Clinical Laboratory Standards. ${ }^{19}$ Further inclusion criteria were adult patients (age $\geq 14$ years) and patients with first or new diagnosis with P.aeruginosa. Patients transferred from another care facility or readmitted to ICU with confirmed diagnosis of P.aeruginosa were excluded.

\section{Controls}

For each case, one control (1:1 basis) who was negative for $P$. aeruginosa infection (i.e.; negative P.aeruginosa patients irrespective of infection) was randomly selected from patients admitted during study period (the same case admission stay, or during the case ICU stay) and same ICU ward.

\section{Data collection}

Data were collected from the hospital microbiology laboratory and cross-checked with hospital computerize databases. Further data were collected on demographic characteristics (age and sex), underlying disorders, cause of hospitalization, ICU stay duration, history of hospitalization, invasive procedures (Foley catheter, naso-gastro tube feeding , mechanical ventilation and tracheostomy and intracentral line), past surgery (during last year), chronic medical conditions (diabetes, blood hypertension, hypothyroidism, congestive heart failure, coronary artery disease, malignancy), dialysis, history of antibiotic therapy and patient's clinical outcome (discharge or death). Reason for medical ICU admission was also captured and classified as burn, cardiac-condition, hematology, kidney disease, medical care, oncology, respiratory disease and trauma.

\section{Antibiotics susceptibility, resistance and intermediate response testing}

Antimicrobial susceptibility testing for P.aeruginosa isolates was performed using Kirby Bauer disk diffusion method as recommended by The USA National Committee for Clinical Laboratory Standards. ${ }^{19}$ Antibiotics drugs tested were: aminoglycosides: amikacin and gentamicin; carbapenem: imipenem/cilastatin and meropenem; cephalosporin: cefepime, ceftazidime, glycylcyclin: tigacyclin, quinolones: ciprofloxacin, penicillins: piperacillin/tazobactam.

\section{Statistical methods}


Data were presented as proportions, means (standard deviation) or medians (interquartile range). The student t-test, Chi-square test (or Fisher exact test) and Mann-Whitney tests were used to compare data; as appropriate. Reason for medical ICU admission was also captured and classified as burn, cardiaccondition, hematology, kidney disease, medical care, oncology, respiratory disease and trauma. Conditional logistic regression models were fitted to identify factors associated with acquisition of $P$.aeruginosa infection. These included age, sex, underlying disorders, cause of hospitalization, ICU stay duration, history of hospitalization, invasive procedures (Foley catheter, naso-gastro tube feeding , mechanical ventilation and tracheostomy and intra-central line), past surgery (during last year), chronic medical conditions (diabetes, blood hypertension, hypothyroidism, congestive heart failure, coronary artery disease, malignancy), dialysis, history of antibiotic therapy and patient's clinical outcome (discharge or death). Variables found significant in univariate analyses were included in the final multivariate model. All tests were two-sided and a p-value 0.05 was considered significant. Effect estimates were expressed as hazard ratio, with 95\% confidence interval. Statistical analyses were performed using Stata ${ }^{\circledR}$ (Stata Corp LP, Texas, USA Institute Inc., Cary, NC, USA).

\section{Results}

During the study period a total of 90 confirmed cases were identified and included in the study. For each case one control was chosen. Table 1 shows the characteristics of these 90 cases and 90 controls. Cases were older than controls but this was not statistically significant $(p=.44)$. Sex distribution was not significantly different in the two groups; the higher proportion in the two groups was males. Cases had significantly higher mean (SD) ICU days stay compared with controls; 37.66(37.82) and 8.37(8.711) respectively; $p=.001$. Previous history of antimicrobial therapy was significantly higher in cases $73(81.1 \%)$ compared with controls 50(55.6\%); $p<.0001$.

Several baseline clinical variables differed in the two groups and were significantly higher in cases compared with controls. These were coronary artery disease $(p=.013)$, malignancy $(p=.036)$, hemodialysis $(p=.004)$, previous surgery $(p<.0001)$, use of central line $(p<.0001)$, urethral catheterization $(p=.002)$, nasogastric tube ( $p<.0001)$, tracheostomy $(p<.0001)$. Mortality was high in both groups. In cases, $54(60 \%)$ patients died compared with $51(56.7 \%)$ in controls; $p=.650$.

In regard to previous use of antimicrobials of the participants in the study, other than tigecycline and penicillins, cases had consistently higher proportion of individuals with history of antimicrobial use. This include cephalosporin $(p=.003)$, aminoglycoside $(p<.0001)$, carbapenem class $(p<.0001)$ and quinolones $(p=.001)$, Table 2 .

Table 3 shows drugs sensitivity and resistance profile of various antimicrobial drugs used for treatment of cases diagnosed with p.aeruginosa. Gentamicin and amikacin were equally documented as being highest sensitive; in 78(86.7\%) patients. A high proportion of number of patients in this group elicited sensitivity to other agents such as cefepime [76(84.4\%)], ceftazidime [70(77.8\%)], ciprofloxacin 
[64(71.1\%)] and piperacillin tazobactam [64(71.1\%)] patients. Tigecycline was the least sensitive $[6(6.7 \%)]$.

A high proportion ( $>90 \%$ ) of resistance was calculated in patients receiving tigecycline [84(93.3\%)]. The least resistance was calculated in those receiving cefepime [8(8.9\%)]. Resistance in other drugs ranged between $12.2 \%$ (gentamicin) to $41.1 \%$ (imipenem).

In contrast, very few patients produced an intermediate reaction to antimicrobial drugs which included meropenem (13.3\%), piperacillin/tazobactam (10\%), ceftazidime (7.8\%) and cefepime (6.7\%). No case was identified with intermediate response to amikacin, imipenem and tigecycline.

The largest percentage of the samples obtained to confirm the diagnosed of $p$.aeruginosa were respiratory culture; $54(60 \%)$. Other culture types were urine [12(13.3\%)], wound specimen [11(12.2\%)], blood [9(10\%)] and fluid [4(4.4\%)].

A conditional logistic analysis was conducted to identify factors associated with $p$. aeruginosa infection (Table 4). Univariate analyses included all patient characteristics, such as demographic, comorbid conditions, invasive diagnostic procedures, history of antibiotic drugs use, previous medical history. Of these characteristics, some were significant $(p<0.05)$ in univariate analyses. These include ICU duration, previous surgery, hemodialysis, tracheostomy, urethral catheterization, aminoglycoside class, carbapenem class and cephalosporins class.

However, in the final multivariate model, factors independently associated with $p$.aeruginosa were ICU duration [Odds Ratio $(\mathrm{OR})=9.05,95 \% \mathrm{Cl} 2.53-32.27, \mathrm{p}=.001$ ], previous surgery $(\mathrm{OR}=7.33 ; 95 \% \mathrm{Cl} 1.66-32.36$, $\mathrm{p}=.009)$, tracheostomy $(\mathrm{OR}=11.13,95 \% \mathrm{Cl} 1.05-118.59, .046)$, urethral catheterization $(\mathrm{OR}=7.38,95 \% \mathrm{Cl}$ $1.21-45.11, \mathrm{p}=.030)$ and aminoglycoside class $(\mathrm{OR}=10.59,95 \% \mathrm{Cl} 1.14-98.13, \mathrm{p}=.038)$.

\section{Discussion}

In this case-control study risk factors, antimicrobial susceptibility pattern, and patient outcomes of $P$.aeruginosa infection were investigated in one of the largest tertiary hospitals in Saudi Arabia. This is the first study in the Gulf countries that assessed these important aspects of $P$. aeruginosa infection in patients managed in the ICUs. This study identifies major causes of nosocomial onset of this infection in patients admitted to ICUs that have important implications for optimal control and prevention.

A significant difference was noted in terms of length of hospital ICU stay in both groups. There was a noticeable increase of number of ICU days among cases with P.aeruginosa compared with controls. In terms of age, male patients in middle to late adulthood age were the ones who frequently acquire this infection. Other studies have shown that age is an important risk factor for of $P$.aeruginosa infection. This nosocomial infection is likewise predominant among male patients as has been reported in previous studies. ${ }^{20,21,32,33}$. 
Hypertension and diabetes mellitus were common among our patients. However, neither of these conditions were significantly linked to P.aeruginosa infection. The occurrence of $P$.aeruginosa is often detected upon the isolation of the pathogenic agent on the patient's fluid and secretions. In the present study, majority of the infection was cultured through respiratory (60\%) discharges with few specimens derived from urine $(13 \%)$, wound $(12 \%)$, blood or fluids of the patient. Comparable with other research works, the site of colonization was often identified in respiratory system, urinary tract, wound and blood. ${ }^{22}$ On other hands, some other studies detected higher rate of P.aeruginosa in urine cultures. ${ }^{23}$

This study found several factors which were associated with development of $P$.aeruginosa. This result concurs with several studies which also aimed at investigating variables that are potential risk factors for P.aeruginosa infection. $9,17,22,24,25$ One of the major findings of this study supports the suggestion that prior utilization of antimicrobial medications is an important predisposing factor for $P$.aeruginos a infection. Patients with previous therapy of aminoglycosides, specifically gentamicin, had a remarkable increased risk of $P$.aeruginosa infection of almost eleven times higher than those who did not use this class of antimicrobial drug. This association persisted after adjustments made in the final multivariate analysis to control for antecedent and concurrent intake of other classes of antimicrobials such as cephalosporins and carbapenem. In similar studies, patient's exposure to antimicrobials before their current hospitalization was a significant predictor of occurrence of P.aeruginosa infection ${ }^{24}$ Although some studies found significant association with former antimicrobial treatments, researchers found that cephalosporins were associated with higher risk than other classes of antibiotics. ${ }^{24}$

A study on antimicrobial resistance patterns previously conducted in this institution, found that P.aeruginosa has a strong tendency to become a multidrug-resistant pathogen. However susceptibility significantly declined after 2007, especially for carbapenem, ceftazidime and ciprofloxacin . ${ }^{14}$ The present study identified large proportion of the samples who were resistant to ampicillin sulbactam, trimethoprim/sulfa and tigecycline.

Furthermore, patients who underwent surgery during the previous year were more likely to become infected with P.aeruginosa than those who did not had any form of operation. P.aeruginosa was seven times higher in this group of patients. However, published research that document proven existence of such association is limited. ${ }^{26}$ Therefore, more studies are needed to confirm this association.

Another important factor attributed to P.aeruginosa colonization was indwelling devices in the patient's body. Patients underwent tracheostomy had approximately eleven times higher rate of P.aeruginosa occurrence than those without tracheal intubation. Similar association was observed in patients who had urethral catheterization. In agreement with this result, some studies found that contraptions were significant determinants of $P$.aeruginosa infection.$^{24,7}$ Some similar published research studies found significant association but no multivariate analysis was conducted. ${ }^{22,27}$ In one study, patients with tracheostomy had increased frequency of respiratory failure; a condition that often caused by 
P.aeruginosa infection. ${ }^{28}$ Another study showed that indwelling devices, such as tracheostomy tubes and urinary catheters, were associated with nosocomial infections. ${ }^{29}$

This study has several limitations that should be acknowledged. This was a retrospective study. Usual limitations of this study design in terms of confirming causality and data quality and comprehensiveness apply. In addition, the study was conducted in a single healthcare center. This has obvious generalizability limitation to other healthcare centers. However, the same protocol in detecting the presence of infection was followed.

\section{Conclusion}

In conclusion, the findings of this study identify the major causes of nosocomial onset of this infection in patients admitted to ICUs. Most of these causes are modifiable and should be aggressively targeted. The findings of this study are of paramount relevance for case identification, control measures, and optimal treatment strategies of P.aeruginosa infection in ICUs.

\section{Abbreviations}

\section{P.aeruginosa}

Pseudomonas aeruginosa

ICU

Intensive care unit

WHO

World Health Organization

MDR

multi-drug resistant

KAMC

King Abdulaziz Medical City

MNG

Ministry of National Guard

AICU

Adult Intensive Care Unit 
NCCU

Neuro Critical Care Unit

BICU

Burn Intensive Care Unit

TICU

Trauma Intensive Care Unit

$\mathrm{MCICU}$

Medical Cardiac Intensive Care Unit

SICU

Surgical Intensive Care Unit

AICU2

Adult Intensive Care Unit-Oncology

\section{Declarations}

Competing interest: None is declared. Funding: This research work was not funded. Authors' contributions: FAA, MB and FAAE conceptualized and designed the study. SA participated significantly in data acquisition. FAA performed the statistical analysis under the supervision FAA and drafted the final manuscript. All authors contributed to revision of final manuscript and approved submission.

Acknowledgment: Not Applicable Ethics and Consent to participate: The study was approved by the Medical Ethics Research Board of King Abdulla International Medical Research Centre. All patients provided written consent for participating in the study.

\section{References}

1. Rello J, Chastre J. Update in pulmonary infections 2012. Am J Respir Crit Care Med 2013; 187:10611066. 2. Vincent JL, Sakr Y, Sprung CL, et al. Sepsis Occurrence in Acutely III Patients Investigators: Sepsis in European intensive care units: Results of the SOAP study. Crit Care Med. 2006; 34:344-353. 3. Sonmezer MC, Ertem G, Erdinc FS, et al. Evaluation of Risk Factors for Antibiotic Resistance in Patients with Nosocomial Infections Caused by Pseudomonas aeruginosa. Can J Infect Dis Med Microbiol. 2016:doi.org/10.1155/2016/1321487. 4. Vitkauskienė A, Skrodenienė E, Dambrauskienè A, Macas A, Sakalauskas R. Pseudomonas aeruginosa bacteremia: resistance to antibiotics, risk factors, and patient mortality. Medicina (Kaunas).2010; 46(7): 490-495. 5. Pinghui VL. Extracellular Toxins of Pseudomonas 
aeruginosa. J Infect Dis.1974; 130|: S94-S99. 6. World Health Organization, (2017). WHO publisheslist of bacteria for which new antibiotics are urgently needed.

http://www.who.int/medicines/publications/WHO-PPL-Short_Summary_25Feb-ET_NM_WHO.pdf/. Accessed 24 January 2019. 7. Ghibu L, Miftode E, Teodor A, Bejan C, Dorobat, CM. Risks factors for Pseudomonas aeruginosa infection, resistant to carbepenem. Rev Med Chir Soc Med Nat lasi.2010; 114(4):1012-6. 8. Santhi M, Sekar U. Multidrug resistant Pseudomonas aeruginosa and Acinetobacter baumannii infections among hospitalized patients: risk factors and outcomes. J Assoc Physicians India. 2009; 57:636-645. 9. Kollef MH, Chastre J, Fagon J et al. Global Prospective Epidemiologic and Surveillance Study of Ventilator-Associated Pneumonia due to Pseudomonas aeruginosa. Crit Care Med. 2014; 42: 2178-2187. 10. Alkeshan YM, Alharbi S, Alrehaili F, Almutiri J, Althobity M, Alotaibi N. Antimicrobial Resistance Pattern of Pseudomonas aeruginosa in Regional Tertiary Care Hospitals of Saudi Arabia. IOSR Journal of Nursing and Health Science. 2016 (5):54-62 . 11. Al-Otaibi FE, Bukhari EE., Badr M, Alraabiaa AA. Prevalence and risk factors of Gram-negative bacilli causing blood stream infection in patients with malignancy. Saudi Med J.2016;37(9), 979-984. 12. Rotimi VO, Alsweih NA, Feteih J. The prevalence and antibiotic susceptibility pattern of gram negative bacterial isolates in two ICUs in Saudi Arabia and Kuwait. Diagn Microbiol Infect Dis1998: 30: 53-59. 13. Asghar AH. Antimicrobial susceptibility and metallo- $\beta$-lactamase production among Pseudomonas aeruginosa isolated from Makkah Hospitals. Pak J Med Sci. 2012;28(5):781-786. 14. Al Johani SM, Akhter J, Balkhy $\mathrm{H}$, El-Saed A, Younan M, Memish Z. Prevalence of antimicrobial resistance among gram-negative isolates in an adult intensive care unit at a tertiary care center in Saudi Arabia. Ann Saudi Med . 2010; (5):364-9. 15. Bauer, K., West, J., O'Brien, J., \& Golf, D. Extended-infusion Cefepine reduces mortality in patients with Pseudomonas aeruginosa infections. Antimicrob Agents Chemother.2013; 57(7), 2907-2912. 16. Nathwani, D., Raman, G., Sulham, K., Gavagham, M., \& Menon, V. Clinical and economic consequences of hospital-acquired resistant and multidrug-resistant Pseudomonas aeruginosa infections: a systematic review and meta-analysis. Antimicrob Resist Infect Control. 2014; 3 (32). 17. Bou, R., Lorente, L., Aguilar, A., Perpinan, J., Ramos, P., Peris, M. \& Gonzales, D. Hospital economic impact of an outbreak in Pseudomonas aeruginosa infection. J Hosp Infect. 2009; 71(2), 138-142. 18. Lautenbach E, Patel JB , Bilker WB, Edelstein PH, Fishman NO Extended-spectrum beta-lactamase-producing Escherichia coli and Klebsiella pneumoniae: risk factors for infection and impact of resistance on outcomes,Clin Infect Dis. 2007; 32: 1162-71. 19. National Committee for Clinical Laboratory Standards: Performance standards for antimicrobial testing. NCCLS, Wayne PAS edition. 2001. pp. Suppl M100-S11. 20. Al-Hasan M, Wilson J, Lahr B, Eckel-Passow J, Baddour L. Incidence of Pseudomonas aeruginosa bacteremia: A populationbased study. AM J Med. 2008; 121(8): 702-708. 21. Venierah AG, Gruson D, Lavigne T, Jarno P, L'heriteau F, Coignard B, Savey A, Rogues A. Identifying new risk factors for Pseudomonas aeruginosa pneumonia in intensive care units: experience of the French national surveillance, REA-RAISIN. J Hosp Infect. 2011; 79(1), 44-48. 22. Shanthi, M. \& Sekar. , aeruginosa and Acinetobacter baumannii infections among hospitalized patients: risk factors and outcomes. J Assoc Physicians India. Sep;57:636, 638-40, 645. U. (2009). Multi-drug resistant 23. Araoka H, Kimura M, Abe M, Takahashi N, Yoneyama, A, Appropriate sampling sites for the surveillance of multidrug-resistant pseudomonas aeruginosa colonization. Jpn J Infect Dis. 2014; 67 (2), 118-189. 24. Aloush, V., Navon-Venezia, S., Seigman-Igra, Y., Cabililand, S. \& 
Carmeli, Y. (2006). Multi-drug-resistant pseudomonas aeruginosa: risk factors and clinical impact. Antimicrob Agents Chemother . 2006; 50(1), 43-48. 25. PengY, Bi J, Shi J, Li Y, Ye X, ChenX, Yao Z. Multidrug resistant pseudomonas aeruginosa infections pose growing threat to health care-associated infection control in the hospitals of Southern China: A case-control surveillance study. Am J Infect Control. 2014; 42(12), 1308-1311. 26. Siripassorn K, Santiprasitkul S, Udompanthurak S, Thamlikitkul V. Risk factors for Pseudomonas aeruginosa bacteremia in Thai patients. J Med Assoc Thai. 2002;85(10):1095-9. 27. Valles J, Mariscal D, Cortes P, Coll P, Villagra A, Diaz E, Artigas A, Rello J. Patterns of colonization by pseudomonas aeruginosa in intubated patients: a 3-year prospective study of 1,607 isolates using pulsed-field gel electrophoresis with implications for prevention of ventilator-associated pneumonia. Intensive Care Med. 2004;, 30(9), 1768-1775. 28. Pignatti, P, Balestrino A, Herr C, Bals R, Moretto D, Corradi M, Alinovi R, Delmastro M, Vogelmeier C, Nava S, Moscato G, Balbi B. Trachesotomy and related host-pathogen interaction are associated with airway inflammation as characterized by tracheal aspirate analysis. Respir Med. 2009; 103 (2), 201-208. 29. Zorgani A, Abofayed A, Glia A, Albarbar A, Hanish S. Prevalence of device-associated nosocomial infections caused by Gram-negative bacteria in a trauma intensive care unit in Libya. Oman Med J. 2015; 30(4), 270-275.

\section{Tables}

Due to technical limitations, table are only available as a download in the supplemental files section

\section{Supplementary Files}

This is a list of supplementary files associated with this preprint. Click to download.

- tables.docx

- INFDD1901113STROBEchecklistcasecontrol1.doc 